[27] G. L. Khodak, "The connection between the redundancy and the delay for VB-encoding," in Proc. III Conf. Theoret. Cybern., Novosibirsk, 1969, pp. 79-80.

[28] ㄴ , "The estimation of redundancy for coding the messages generated by a Bernoulli source," Próbl. Inform. Trans., vol. 8, no. 2, pp. 28-32, 1972.

[29] - "Coding of Markov sources with low redundancy," in Proc. 2nd Int. Symp. Inform. Thenry, Tsahkadzor, Acad. Kiado, Budapest, 1973, pp. 201-209.

[30] - "The cost of transmission of sequences," Probl. Inform. Contr., vol. 4, no. 4, pp. 299-311, 1975.

[31] I. Csizăr, "On noiseless channels," Probl. Inform. Trans., vol. 1, no. 4, pp. 3-15, 1970

[32] Yu. M. Starkov, "The coding of finite messages on output of a source with unknown statistic," in Proc. V Conf. Inform. Theory, Moscow-Gorkii, 1972, pp. 147-152.

[33] Yu. M. Starkov and V. F. Babkin, "Combinatorial encoding for discrete stationary sources," in Proc. 2nd Int. Symp. Inform. Theory, Tsahkadzor, Armenia, USSR, 1971; Academia Kiado, Budapest, 249-257, 1973.

[34] T. M. Cover, "Admissibility properties of Gilbert's encoding for unknown source probabilities. IEEE Trans. Inform. Theory, vol. 18, no. 1, pp. $216-217,1972$.

[35] T. M. Cover, "Enumerative sources encoding," IEEE Trans. Inform. Theory, vol. 1-19, no. 1, pp. 73-77, 1973.

[36] L. D. Davisson, "Comments on 'Sequence time coding for data compression," Proc. IEEE, vol. 54, p. 2010, Dec. 1966.

[37] L. D. Davisson, "Universal noiseless coding," IEEE Trans. Inform. Theory, vol. 19, no. 6, pp. 783-795 1973.

[38] P. Elias, "Universal codeword sets and representations of the integers," IEEE Trans. Inform. Theory, vol. 21, no. 2, pp. 194-203, 1975.
[39] R. M. Fano, Transmission of Information. Cambridge, MA: MIT Press and New York: Wiley, 1961.

[40] W. Feller, An Introduction to Probability Theory and its Applications, vol. 2. New York: Wiley, 1966.

[41] R. M. Gray and L. D. Davisson, "Source coding theorems without the ergodic assumption," IEEE Trans. Inform. Theory, vol. 20, no. 4, pp. $509-510,1979$.

[42] R. M. Gray and L. D. Davisson, "The ergodic decomposition of stationary discrete random processes," IEEE Trans. Inform. Theory, vol. 20 , no. 5 , pp. $625-636,1974$

[43] E. N. Gilbcrt, "Coding based on inaccurate source probabilities," IEEE Trans. Inform. Theory, vol. 17, no. 3, pp. 304-314, 1971.

[44] F. Jelinek and K. Schneider, "On variable-length to block coding," IEEE Trans. Inform. Theory, vol. 18, no. 6, pp.765-774, 1977.

[45] T. J. Lynch, "Sequence time coding for data compression," Proc. IEEE, vol. 54, no. 10, pp. 1490-1491, 1966.

[46] J. C. Lawrence, "A new universal coding scheme for coding the binary memoryless source," IEEE Trans. Inform. Theory, vol. 23, no. 4 , pp. 446-472, 1977.

[47] B. McMillan, "Two inequalities implied by unique decipherability," IRE Trans. Inform. Theory, vol.2, no. 4, pp. 115-116, 1956.

[48] D. L. Neuhoff, R. M. Gray, and L. D. Davisson, "Fixed rate universal block source coding with fidelity criterion," IEEE Trans. Inform. Theory, vol. 21, no. 5, pp. 511-513, 1975.

[49] C. E. Shannon, "Mathematical theory of communication," Bell Syst. Tech.J., vol. 27 , no. 3 , pp. $379-423$, pp. $623-656,1978$.

[50] J. Ziv, "Coding of sources with unknown statistics-Part 1: Probability of encoding error," IEEE Trans. Inform. Theory, vol. 18, no. 3, pp. 384-389, 1972.

[51] _ "Coding of sources with unknown statistics-Part II: Distortion relative to fidelity criterion," IEEE Trans. Inform. Theory, vol. 18, no. 3, pp. 389-394, 1972.

\title{
Companding and Random Quantization in Several Dimensions
}

\author{
JAMES A. BUCKLEW, MEMBER, IEEE
}

\begin{abstract}
The problem of implementing multidimensional quantizers is discussed. A general equation is derived that can be used to evaluate the performance of multidimensional compandors. It is demonstrated that the optimal compandor must be conformal almost everywhere. An example is given to show that asymptotically optimal performance could be obtained through nonconformal companding schemes. Random quantizers are discussed and two techniques are evaluated for reducing memory and computation time in the implementation of such devices.
\end{abstract}

Manuscript received February 10, 1980; revised May 5, 1980. This paper was presented at the 1980 Princeton Conference on Information Sciences and Systems, Princeton, NJ, March 1980.

The author is with the Department of Electrical and Computer Engineering, University of Wisconsin, Madison, WI 53705.

\section{INTRODUCTION}

$\mathrm{T}$ HE IMPLEMENTATION of high-dimensional block quantizers has in the past received relatively little attention. In this paper we put forth two techniques, companding and random quantization, to solve this problem.

W. R. Bennett [1] was the first to model a nonuniform quantizer as a zero-memory nonlinearity followed by a uniform quantizer, in turn followed by the inverse of the first nonlinearity. This sequence of operations is generally referred to as companding. The word expresses the idea that the data is first compressed, then quantized, and then expanded. The first nonlinearity is therefore generally re- 
ferred to as the "compressor" and its inverse as the "expander".

Compandors are of interest in areas other than quantization theory. In some analog systems it can be advantageous to use compandors because of signal dynamic range considerations. Analog music signals are sometimes passed through a nonlinearity that reduces the range of the large signal excursions while amplifying the small signal portions. Before playback the signal is passed through the inverse nonlinearity which tends to reduce the noise in the low signal levels. Respectable gains in the signal-to-noise ratio can be accomplished with such schemes.

The third section of this paper is an investigation of companding in several dimensions. In several dimensions the compressor characteristic is a mapping function $f$ : $\mathbb{R}^{k} \rightarrow X_{i=1}^{k}(0,1)$, where $X$ denotes the Cartesian cross product and $X_{i=1}^{k}(0,1)$ is a $k$-dimensional hypercube. In the companding approach to optimal quantization, the quantizer output levels are distributed in the hypercube. We usually choose from these output levels the nearest neighbor to $f(\boldsymbol{x})$, where $\boldsymbol{x}$ is the input data vector. The quantized output is then $f^{-1}$ of this particular output level.

The theory will also hold for analog signal processing in several dimensions. It does not matter whether the noise is quantization noise or any other kind of additive noise as long as the noise components in each channel are uncorrelated with one another. For example, let us denote the error vector caused by quantization in the hypercube by $\left(r_{1}, r_{2}, \cdots, r_{k}\right)^{T}$. Then the condition that is needed is $E\left\{r_{i} r_{j}\right\}$ $=\sigma_{r}^{2} \delta_{i j}$, where $\delta_{i j}$ is the Kronecker delta function. In a practical sense, this assumption is not very restrictive. It may be shown, at least asymptotically (as the number of output levels in the hypercube approaches infinity), that the error vector in an optimal or random quantizer converges to an hyperspherically symmetric probability density which satisfies our above condition.

The fourth section of this paper will deal with various techniques for implementing high-dimensional random quantizers. A random quantizer is one where the output levels are samples from some $k$-dimensional probability distribution $\lambda(\boldsymbol{x})$. If a companding approach is desired, then $\lambda(x)$ would be defined on $X_{i=1}^{k}(0,1)$. It is known that random quantizers (with the correct $\lambda(\boldsymbol{x})$ ) approach the optimum quantizer performance as $k$ approaches infinity. The implementation schemes discussed in this section are essentially search algorithms for quantizer output levels which enable us to find a "good" output level for a particular data vector.

\section{Previous Work}

Many authors have considered the problem of designing an optimal quantizer subject to some difference distortion measure. Max [2] gives necessary but not sufficient conditions for the optimal one-dimensional quantizer, and Fleisher [3] provides a sufficient condition that requires certain convexity properties of the density function of the input random variable.
Panter and Dite [4] derive an expression for the expected mean square error of a minimum mean square error onedimensional quantizer, assuming the number of output levels to be very large. Algazi [5] generalizes Panter and Dite's equation to a $t$ th power distortion measure. Wood [6] uses some equations derived by Roe [7] to rederive Panter and Dite's result and give formulas for obtaining the asymptotic quantizer's output levels. Zador [8] generalizes the work of Panter and Dite to several dimensions and to a more general difference distortion measure. Zador's equation for the distortion error is

$$
C(k, t) N^{-t / k}\|p\|_{k /(k+t)}=\frac{1}{k} E\left\{\|x-Q(x)\|_{2}^{t}\right\},
$$

where

$\begin{array}{ll}\boldsymbol{x} & \text { input random vector } \\ Q(\boldsymbol{x}) & \text { quantized random vector } \\ E\{\cdot\} & \text { statistical expectation operator } \\ N & \text { number of output levels and assumed to be large } \\ k & \text { dimension of } \boldsymbol{x} \\ C(k, t) & \text { constant depending only upon } k \text { and } t \\ p(\boldsymbol{x}) & \text { probability density of } \boldsymbol{x} \\ \|p\|_{\alpha} & {\left[\int p(\boldsymbol{x})^{\alpha} d_{x_{1}} \cdots d_{x_{k}}\right]^{1 / \alpha} .}\end{array}$

Zador also shows that $\lim _{k \rightarrow \infty} C(k, 2)=1 / 2 \pi e . C(1,2)$ is known to be $1 / 12$. Gersho [9] gives an alternate derivation of the above equation and derives new bounds for $C(k, t)$, which is known for only a few values of $k$ and $t$. Yamada, et al. [10], extend Zador's work to more general cost functions of the error.

\section{COMPANDOR ERROR Derivation}

Our data will be assumed to be $k$-dimensional samples from a probability density function $p(x), x \in \mathbb{R}^{k}$. Let $D_{p}$ be the support of $p(x)$. Let $f: D_{p} \rightarrow X_{i=1}^{k}(0,1)$ be regular and onto.

We force $f$ to be onto; if it were not there would be code vectors in the hypercube that would never be used, and hence the quantizer would be suboptimal. We use this condition at only one point in the derivation as a constraint on the optimal compandor. All equations derived up to that point are valid without this restriction. We will sometimes represent the mapping by

$$
f=\left(f_{1}(x), f_{2}(\boldsymbol{x}), \cdots, f_{k}(\boldsymbol{x})\right)^{T} .
$$

Let $\boldsymbol{r}=\left(r_{1}, r_{2}, \cdots, r_{k}\right)^{T}$ be the error vector in the hypercube. As stated before, under some fairly general conditions $E\left\{r_{i} r_{j}\right\}=\left(E\left\{r^{2}\right\} \delta_{i j}\right) / k$ where $\delta_{i j}$ is the Kronecker delta. Assuming very small distortion, a good approximation to the final error vector in the output is $\left(f^{-1}\right)^{\prime}(y) r$. When $k=1$ this is equivalent to $f(x+\Delta x) \simeq f^{\prime}(x) \Delta x+f(x)$. Let $\boldsymbol{y}$ be the variable in the hypercube. If $\boldsymbol{y}=\boldsymbol{f}(\boldsymbol{x})$, then

$$
p_{y}(\boldsymbol{y})=\frac{p_{x}\left(f^{-1}(\boldsymbol{y})\right)}{\left|f^{\prime}\left(f^{-1}(\boldsymbol{y})\right)\right|}
$$

where $|\cdot|$ indicates determinant. Therefore the mean square 
error (mse) of the final output may be written

$$
\begin{aligned}
\mathrm{mse}=\int_{X_{i=1}^{k}(0,1)} \boldsymbol{r}^{T}\left(f^{-1}\right)^{\prime T}\left(f^{-1}(y)\right) \\
\cdot\left(f^{-1}\right)^{\prime}\left(f^{-1}(y)\right) r \frac{p_{x}\left(f^{-1}(y)\right) d y}{\left|f^{\prime}\left(f^{-1}(y)\right)\right|} .
\end{aligned}
$$

Let $\boldsymbol{x}=f^{-1}(\boldsymbol{y})$. Then $d \boldsymbol{x}=\left|\left(f^{-1}\right)^{\prime}(y)\right| d \boldsymbol{y}$ and $\left|\left(f^{-1}\right)^{\prime}(\boldsymbol{y})\right|$ $=\left(\left|f^{\prime}\left(f^{-1}(y)\right)\right|\right)^{-1}$ by the inverse mapping theorem [11]. Making these changes of variables, we obtain

$$
\text { mse }=\int_{D_{p}} \boldsymbol{r}^{T}\left[f^{\prime}(\boldsymbol{x})\right]^{-1 T}\left[f^{\prime}(\boldsymbol{x})\right]^{-1} \boldsymbol{r} p_{x}(\boldsymbol{x}) d \boldsymbol{x},
$$

again by the inverse mapping theorem. Write $\left[f^{\prime}(\boldsymbol{x})\right]^{-1 T}\left[f^{\prime}(\boldsymbol{x})\right]^{-1}=\Sigma^{-1}(\boldsymbol{x})$, which is a symmetric matrix for every $x$. Therefore our problem is to optimize

$$
\int_{D_{p}} \boldsymbol{r}^{T} \Sigma^{-1}(\boldsymbol{x}) r p_{x}(\boldsymbol{x}) d x
$$

Using a matrix identity, we can restate the above integral as

$$
\int_{D_{p}} \operatorname{tr}\left\{\Sigma^{-1}(\boldsymbol{x}) \boldsymbol{r} r^{T}\right\} p_{x}(\boldsymbol{x}) d x
$$

Let us take the expectation over the $r$ variable which is independent of any other quantity in the integral. (We can make a random coding argument to insure that the $r$ variable be independent, although it is tedious to do so.)

$$
E\left\{r r^{T}\right\}=E\left\{\left(\begin{array}{cccc}
r_{1}^{2} & r_{1} r_{2} & \cdots & r_{1} r_{n} \\
r_{2} r_{1} & r_{2}^{2} & & r_{2} r_{n} \\
r_{n} r_{1} & \cdots & \cdots & r_{1}^{2}
\end{array}\right)\right\}=\frac{E\left\{r^{2}\right\}}{k} I .
$$

Therefore,

$$
\text { mse }=\frac{E\left\{r^{2}\right\}}{k} \int_{D_{p}} \operatorname{tr}\left\{\Sigma^{-1}(x)\right\} p_{x}(x) d x .
$$

This expression is of interest in its own right. $E\left\{r^{2}\right\} / k$ is the mean square error per sample suffered by the hypercube quantization. The total error is therefore a product of two independent terms. Denote the eigenvalues of $\Sigma(x)$ by $\lambda_{i}^{2}(\boldsymbol{x})(i=1, \cdots, k)$. Then

$$
\text { mse }=\frac{E\left\{r^{2}\right\}}{k} \sum_{i=1}^{k} \int \frac{p_{x}(\boldsymbol{x})}{\lambda_{i}^{2}(\boldsymbol{x})} d \boldsymbol{x} .
$$

Since our map $f$ is onto, we have

$$
\int_{D_{p}}\left|f^{\prime}(x)\right| d x=\int \prod_{i=1}^{k} \lambda_{i}(x) d x=1 .
$$

Let us minimize the mse subject to the above constraint. First, it is easy to show that $\lambda_{i}(x)=\lambda(x)$ for every $i$. So we must minimize

$$
\int_{D_{p}} \frac{p(\boldsymbol{x})}{\lambda(\boldsymbol{x})^{2}} d \boldsymbol{x}
$$

subject to the constraint

$$
\int_{\mathbb{R}^{k}} \lambda(x)^{k} d x=1
$$

Writing $\beta(x)=\lambda(x)^{k}$, we must minimize

$$
\int \frac{p(x)}{\beta(x)^{2 / k}} d x, \quad \text { where } \quad \int \beta(x) d x=1 .
$$

Gersho [9] shows that the optimal $\beta(x)$ is proportional to $p(\boldsymbol{x})^{1 /(I+2 / k)}=p^{k /(k+2)}(\boldsymbol{x})$, which implies that $\lambda(\boldsymbol{x})=$ $p(\boldsymbol{x})^{1 /(k+2)} /\left(\|p\|_{k /(k+2)}\right)^{1 /(k+2)}$. Using these eigenvalues, we can set the mse $=E\left\{r^{2}\right\} k^{-1}\|p\|_{k /(k+2)}$. If an optimal $k$-dimensional uniform quantizer is implemented in the hypercube, then this equation gives the same error as Zador's optimum quantizer. The condition for the optimal compressor is that all the eigenvalues of the symmetric matrix $\Sigma(x)=\left[f^{\prime}(x)\right]\left[f^{\prime}(x)\right]^{T}$ be the same. This condition implies that there exists an orthonormal matrix $\phi(x)$ such that $\phi^{T}(\boldsymbol{x}) \Sigma(\boldsymbol{x}) \phi(\boldsymbol{x})=\lambda^{2}(\boldsymbol{x}) I ;$ or $\Sigma(\boldsymbol{x})=\lambda^{2}(\boldsymbol{x}) I=$ $\left[f^{\prime}(\boldsymbol{x})\right]\left[f^{\prime}(\boldsymbol{x})\right]^{T}$, which implies that $\left(\left[f^{\prime}(\boldsymbol{x})\right]\right) / \lambda(\boldsymbol{x})$ is an orthonormal matrix. Since $\lambda(\boldsymbol{x})$ is known in principle we could solve for $f^{\prime}(\boldsymbol{x})$ for every value of $\boldsymbol{x}$. Therefore the condition for an optimal compandor is that $\left[f^{\prime}(\boldsymbol{x})\right] / c p(\boldsymbol{x})^{1 /(k+2)}$ be an orthogonal matrix for almost every value of $\boldsymbol{x}$ where $c=1 /\left(\|p\|_{k /(k+2)}\right)^{1 /(k+2)}$.

When $k=2$ this condition says that $f(\boldsymbol{x})$ must be conformal almost everywhere except for a set of measure zero. Gersho points out (for the two-dimensional case) that conformal maps do not exist for circularly symmetric probability densities. An illustration of this fact is the work by Heppes and Szuz [12] which shows it is not possible to tessellate a circular region with an arbitrary "surface distribution function" using regular hexagons. There must always be a "slit" where the tessellation fails. This "slit", however, is a set of measure zero. Only local conformality almost everywhere is needed, not global conformality.

We now provide an example illustrating (2). Suppose that the input probability density $p(x)$ can be written as $\Pi_{i=1}^{k} p\left(x_{i}\right)$. Let $C=1 / \int_{-\infty}^{\infty} p(x)^{\alpha} d x$ and let the compressor function $f=\left(f_{1}\left(x_{1}\right), f_{2}\left(x_{2}\right), \cdots, f_{k}\left(x_{k}\right)\right)^{T}$ where $f_{i}\left(x_{i}\right)=$ $C \int_{-\infty}^{x_{i}} p(x)^{\alpha} d x$. With little loss of generality, we assume $f$ is regular; $f$ is also obviously onto. Hence,

$\left[f^{\prime}(\boldsymbol{x})\right]=\left[\begin{array}{cccc}C p\left(x_{1}\right)^{\alpha} & 0 & \cdots & 0 \\ 0 & C p\left(x_{2}\right)^{\alpha} & & \vdots \\ 0 & & \ddots & 0 \\ \vdots & & 0 & C p\left(x_{k}\right)^{\alpha}\end{array}\right]$

$$
\left[f^{\prime}(\boldsymbol{x})\right]^{-1}=\left[\begin{array}{ccccc}
\frac{1}{C p\left(x_{1}\right)^{\alpha}} & & 0 & \ldots & 0 \\
& \ddots & \frac{1}{C p\left(x_{2}\right)^{\alpha}} & & \\
0 & & & \ddots & \\
\vdots & & & 0 & \frac{1}{C p\left(x_{k}\right)^{\alpha}}
\end{array}\right]
$$


The eigenvalues of $\Sigma^{-1}(x)$ are $1 /\left(C^{2} p\left(x_{i}\right)^{2 \alpha}\right), i=1, \cdots, k$, so the error may be written

$$
\begin{aligned}
\mathrm{mse} & =\frac{E\left\{r^{2}\right\}}{k} \sum_{i=1}^{k} \int_{D_{p}} \frac{\prod_{j=1}^{k} p\left(x_{j}\right) d x}{C^{2} P\left(x_{i}\right)^{2 \alpha}} \\
& =\frac{E\left\{r^{2}\right\}}{C^{2}} \int_{-\infty}^{\infty} p(x)^{1-2 \alpha} d x \\
& =E\left\{r^{2}\right\}\left[\int_{-\infty}^{\infty} p(x)^{\alpha} d x\right]^{2}\left[\int_{-\infty}^{\infty} p(x)^{1-2 \alpha} d x\right] .
\end{aligned}
$$

Using Hölders inequality we may show that $\alpha=1 / 3$ minimizes the error:

$$
\mathrm{mse}=E\left\{r^{2}\right\}\|p\|_{1 / 3} \text {. }
$$

But using Zador's coefficient for the one-dimension case (see (1)) we have

$$
\mathrm{mse}_{1-\operatorname{dim}}=\frac{\|p\|_{1 / 3}}{12 N^{2}}
$$

Therefore, this compressor characteristic gives us the same error as the optimal one-dimensional quantizer if in the hypercube we quantize with one-dimensional uniform quantizers. We can quantize in the hypercube using optimal schemes for a coefficient of

$$
\text { mse }=\frac{\|p\|_{1 / 3}}{N^{2} 2 \pi e}, \quad \text { as } K \rightarrow \infty .
$$

Therefore the best we may produce with this compressor characteristic is a gain of $(2 \pi e) / 12 \cong 1.42$ in signal to quantizing noise ratio, at the expense of implementing optimal uniform quantizers in the hypercube.

As a second example, again let $p(\boldsymbol{x})=\prod_{i=1}^{k} p\left(x_{i}\right)$. Suppose we choose the eigenvalues of $\Sigma(x)$ to be

$$
\lambda_{i}^{2}(x)=\left[\frac{\prod_{\substack{j=1 \\ j \neq i}}^{k} p\left(x_{i}\right)^{1 /(k+1)}}{\int_{-\infty}^{\infty} p(x)^{(k-1) /(k+1)} d x}\right]^{2}
$$

which obviously leads to a nonconformal map. Using [2], we find the mean square error for such a compressor characteristic to be

$$
\text { mse }=E\left\{r^{2}\right\}\|p\|_{(k-1) /(k+2)}=E\left\{r^{2}\right\}\|p\|_{(k-1) /(k-1)+2},
$$

which is the optimal coefficient for the $(k-1)$-dimensional space. This relation implies the possibility of obtaining nonconformal mapping functions that will asymptotically give optimal results.

\section{RANDOM QuANTIZATION}

A multidimensional quantizer is essentially a partition of space. If a data sample falls within a certain set in the partition, it is assigned to a particular output level. It is known that the optimal partition of the hypercube consists of polytopes (multidimensional polygons). In $\mathbb{R}^{\prime}$ there is only one possibility, the line segment. In $\mathbb{R}^{2}$ the optimal polytope is the hexagon. In $\mathbb{R}^{3}$ Gersho conjectures that it is the truncated octahedron. The optimal polytope is not known for dimensions greater than three, and hence it seems very difficult to actually design a high-dimensional optimum quantizer. Linde, et al. [13] present an algorithm to design such quantizers that is guaranteed to converge to a local optimum but not always a global optimum. An alternative method for obtaining an asymptotically optimal formulation would be to assign the quantizer's output levels to be random samples from some point density function $\lambda(\boldsymbol{x})$. We would then find the nearest neighbor to our data vector from these values and use that neighbor as our quantized vector. Zador's upper bound to $C(k, t)$ can be derived by considering the error for such a quantizer. Zador and Gersho both point out that as the dimension of the random quantizer becomes very large, its performance approaches that of the optimum quantizer $(\lambda(x)$ must be proportional to $\left.p^{k /(k+2)}(\boldsymbol{x})\right)$. There is a drawback, however, to the straightforward implementation of such a quantizer. For a fixed data rate (say $b$ bits per sample) the number of output levels in the quantizer is an exponential function of $k$ ( $2^{b k}$ in fact). A nearest neighbor search for the closest output level to a particular data vector could require inordinate amounts of computer time.

In this section we will discuss some optimal and suboptimal techniques to find a quantized value. In a search scheme for an optimal nearest neighbor, we can visualize an hypersphere slowly expanding about the data point until the surface of the sphere comes in contact with a quantizer output level. This output level is then the nearest neighbor. To implement such a scheme we must calculate the Euclidean distance between our data vector and every output level. A Euclidean distance calculation is cumbersome in that it requires multiplications ( $K$ per sample). Thus we need to do $N K=2^{b k} K$ multiplications.

An alternate search scheme is to expand a hypercube about the data point until it meets an output level. To find this "largest" hypercube requires only additions. Let us calculate the mean square error of such a scheme.

The probability of an output level being in a hypercube of side length $r$ is $\lambda(x) r^{k}$. Then making use of order statistics, the probability density of the smallest hypercube side out of $N$ samples is $K N\left[1-r^{k} \lambda(x)\right]^{N-1} r^{k-1} \lambda(x)$. Suppose we have searched and found the largest hypercube; there must therefore be an output level somewhere on the surface of that hypercube. If $N$ is large, $p(x)$ varies very little in this largest hypercube, and the output level may be considered to be uniformly distributed on this largest hypercube of side $r$. Since there are $2 k$ sides, the mean square error may then be written (given $r$ ) as

$$
\begin{aligned}
\text { mse } \mid r= & \frac{2^{k-1}}{r^{k-1}} \underbrace{\int_{0}^{r / 2} \cdots \int_{0}^{r / 2}\left[\left(\frac{r}{2}\right)^{2}\right.}_{\begin{array}{c}
k-1 \\
\text { integrals }
\end{array}} \\
& \left.+n_{1}^{2}+n_{2}^{2}+\cdots+n_{k-1}^{2}\right] d n_{1} \cdots d n_{k}=\frac{(k+2) r^{2}}{12}
\end{aligned}
$$


Taking the expectation over $r$, we find that the mean square error is

$$
\begin{aligned}
\frac{k+2}{12} \int r^{2} N\left[1-\lambda(\boldsymbol{x}) r^{k}\right] & N-1 \\
& k r^{k-1} \lambda(x) d r p(x) d x \\
& =\frac{k+2}{12 N^{2 / k}} \Gamma\left(1+\frac{2}{k}\right) \int \frac{p(x)}{\lambda(x)^{2 / k}} d x .
\end{aligned}
$$

$\lambda(x)$ is optimized when it is proportional to $p(x)^{k / k+2}$, and the error becomes

$$
\mathrm{mse}=\frac{k+2}{N^{2 / k} 12} \Gamma\left(1+\frac{2}{k}\right)\|p(x)\|_{k /(k+2)} .
$$

If $k$ is very large then the error becomes

$$
\text { mse per sample }=\frac{\|p\|_{k /(k+2)}}{N^{2 / k} 12} .
$$

(1/12 is the one-dimensional coefficient.) By doing no multiplication, we accept a degradation of $(2 \pi e) / 12$ in signal to quantizing noise ratio over that of the optimal search (see the first example in the previous section).

The hypercube search still requires searching through $N$ code vectors to find the largesi hypercube. We now describe a technique that will allow us to shorten the number of codewords to be searched. Since optimal companding gives optimal performance, we will restrict our discussion to data densities contained in the hypercube. We cut our $k$ dimensional hypercube into several smaller hypercubes. If we slice each edge of the hypercube into $2^{b_{1}}$ equal intervals using $k-1$ dimensional perpendicular hyperplanes, we will have partitioned the large hypercube into $2^{k b_{1}}$ small hypercubes or "cubelettes". In one cubelette we randomly throw $N / 2^{k b_{1}}$ uniformly distributed $k$-dimensional points which will be the quantizer output levels. We then replicate this cubelette through $k$ space $2^{k b_{1}}$ times until we have filled up our original large cube. If we assume that there is a low probability that a data vector will be closer to the edge of a cubelette than to an output level, then we can show that this quantization scheme will have the same error as if we used uniformly distributed output levels through the whole hypercube.

Using one-dimensional uniform quantizers that are extremely easy to implement, we may quickly decide which one of the cubelettes contains the data vector. We then do a sequential search on $N / 2^{k b_{1}}=2^{k\left(b-b_{1}\right)}$ output levels where $k b$ is the number of bits per $k$-dimensional sample.

How large may $b_{1}$ be set? Obviously if $b_{1}=b$ we have one output level per cubelette, so that for a given data vector there is a good chance the edge of the cubelette will be closer then the output level. One way to get an idea of the number of output levels actually needed would be to calculate the probability of hitting an edge before a data point. To make things simpler, suppose that

$$
p(\boldsymbol{x})=\prod_{i=1}^{k} p\left(x_{i}\right)
$$

and that $p(x)$ is symmetric. The probability that the data point component $x_{i}$ is closer to zero or one than to a corresponding output level component $y_{i}$ is $\left|1-2 x_{i}\right|$. If $p(x)$ is symmetric, then $E\left|1-2 x_{i}\right|=1 / 2$. The probability that a data vector is closer to a particular code vector than to an edge is $1 / 2^{k}$. The probability that out of $N$ output levels the data vector is closer to the edge than to any of them is $\left[1-1 / 2^{k}\right]^{N}$. Of course, we want this probability to be very small. If $N=C 2^{k}$, then for large $k$ the probability of hitting an edge before a code vector is $c^{-c} . N$ therefore needs to be on the order of $2^{k}$ for all of our assumptions to hold and hence $b_{1}$ may be set at $b-1$ or slightly smaller.

\section{Summary}

A general expression for the performance of a multidimensional compander was derived and it was proved that the optimal compressor characteristic must be conformal almost everywhere. An example was given that indicates that asymptotically optimal performance may be gained even with nonconformal maps. Random quantizers were discussed which are known to be asymptotically optimal and two techniques to reduce computer time in their implementation were evaluated.

\section{REFERENCES}

[1] W. R. Bennett, "Spectra of quantized signals," Bell. Syst. Tech. J., vol. 27 , pp. 446-472, July 1948.

[2] J. Max, "Quantizing for minimum distortion," IRE Trans. Inform. Theory, vol. IT-6, pp. 7-12, Mar. 1960.

[3] P. E. Fleisher, "Sufficient conditions for achieving minimum distortion in a quantizer," IEEE Int. Conv. Rec., pt. I, pp. 104-111, 1964.

[4] P. F. Panter and W. Dite, "Quantization in pulse-count modulation with nonuniform spacing of levels," Proc. IRE, vol. 39, pp. 44-48, 1951.

[5] V. A. Algazi, "Useful approximations to optimum quantization," IEEE Trans. Commun. Technol, vol. COM-14, pp. 297-301, 1966.

[6] R. C. Wood, "On optimimum quantization," IEEE Trans. Inform. Theory, vol. IT-5, pp. 248-252, Mar. 1969.

[7] G. M. Roe, "Quantizing for minimum distortion," IRE Trans. Inform. Theory, vol. IT-10, pp. 384-385, Oct. 1964.

[8] P. Zador, "Development and Evaluation of Procedures for Quantizing Multivariate Distributions", Ph.D. dissertation, Stanford, Univ. Stanford, CA, 1964, univ, microfilm no. 64-9855.

[9] A: Gersho, "Asymptotically optimal block quantization," IEEE Trans. Inform. Theory, vol. IT-25, pp. 373-380, July 1979.

[10] Y, Yamada, S. Tazaki, and R. M. Gray, "Asymplotic performance of block quantizers with difference distortion measures," IEEE Trans. Inform. Theory, vol. IT-26, pp. 6-14, Jan. 1980.

[11] W. Fleming, Functions of Several Variables. New York: Springer, 1977.

[12] A. Heppes and P. Szüsz, "Bemerkung zu einer Arbeit von L. Fejes Toth," El. Math., vol. 15, pp. 134-136, 1960.

[13] Y. Linde, A. Buzo, and R. M. Gray, "An algorithm for vector quantizcr design," IEEE Trans. Comm., vol. COM 28, pp. 8495 , Jan. 1980. 